\title{
GRAMÍNEAS TROPICALES EN EL ENGORDE DE CUYES MEJORADOS SEXADOS (Cavia porcellus Linnaeus) EN LA ZONA DE LA MANÁ
}

\author{
Adolfo Sánchez Laiño ${ }^{1,2}$, Sixto Sánchez Gallardo², Saúl Godoy Becerra ${ }^{2}$, Raúl Díaz Ocampo y Norma Vega Pastuña ${ }^{2}$ \\ ${ }^{1}$ Unidad de Investigación Científica y Tecnológica, Universidad Técnica Estatal de Quevedo, $\mathrm{km} 1$ 1/2 \\ vía Quevedo - Santo Domingo de los Tsáchilas, C. P. 73. Quevedo, Los Ríos, Ecuador. \\ ${ }^{2}$ Facultad de Ciencias Pecuarias, Universidad Técnica Estatal de Quevedo, $\mathrm{km} 7$ vía a El Empalme \\ Mocache, Los Ríos, Ecuador
}

\section{RESUMEN}

L a investigación de gramíneas tropicales en el engorde de cuyes en la zona de La Maná. La misma que persiguió los siguientes objetivos: Incrementar los índices productivos en cuyes (Cavia porcellus Linnaeus) peruanos mejorados bajo el efecto del consumo del pasto saboya (Panicum maximun Jack), hojas de maíz (Zea mays) y caña de azúcar (Saccharum officinarum L) en la zona de La Maná, y determinar la rentabilidad de los tratamientos. Se utilizaron 48 cuyes peruanos mejorados sexados de 35 días de edad con un peso promedio de 305 g. Se aplicó un arreglo factorial 2 (sexo) x 3 (gramíneas) con cuatro repeticiones, dentro de un (DBCA). Para las comparaciones entre medidas se utilizó la prueba de Tukey al $0.05 \%$ de probabilidad. Para obtener la rentabilidad de los tratamientos se utilizó la Relación Beneficio-Costo. Se evaluó el: consumo de gramíneas $(\mathrm{g})$, consumo de alimento $(\mathrm{g})$, ganancia de peso $(\mathrm{g})$, conversión alimenticia, peso vivo $(\mathrm{g})$ y rendimiento a la canal (\%). Los cuyes machos presentaron el mayor $(\mathrm{P}<0.05)$ consumo de gramínea, ganancia de peso y peso vivo (81.65 $\mathrm{g} \mathrm{día}^{-1}$ de MS; $8.66 \mathrm{~g}_{\text {animal }}{ }^{-1}$ día $^{-1} ; 827.7 \mathrm{~g}$, respectivamente). La gramínea de mayor consumo $(\mathrm{P}<0.05)$ fue el pasto saboya $\left(99.31 \mathrm{~g}^{\text {animal }}{ }^{-1}\right.$ día $^{-1}$ de MS). Sin embargo registró la conversión alimenticia $(\mathrm{P}<0.05)$ menos eficiente $(13.82)$. La mayor ganancia de peso $(\mathrm{P}<0.05)$ la registró el tratamiento en base a hoja de maíz (9.16 $\left.\mathrm{g}_{\text {animal }}{ }^{-1} \mathrm{dí}^{-1}\right)$. El rendimiento a la canal $(\mathrm{P}>0.05)$ no se vio afectado por el efecto del consumo de gramíneas. La mayor rentabilidad la registraron los cuyes machos alimentados con hoja de maíz + balanceado $(52.4 \%)$.

Palabras claves: Gramíneas, forraje, alimentación, cuyes
Abstract

The investigation of tropical gramineous in guinea 1 pigs in the area of La Mana. The following objectives were pursued: To increase the productive indexes in improved peruvian guinea pigs (Cavia porcellus Linnaeus) under the effect of the consumption of the saboya grass (Panicum maximun Jack), leaves of Corn (Zea mays) and cane of sugar (Saccharum officinarum L) in the area of La Mana, and to determine the profitability of the treatments. 48 improved peruvian guinea pigs separated by sex of 35 days of age were used with a weight average of $305 \mathrm{~g}$. A factorial form 2 (sex) x 3 (gramineous) with four replications was applied, arranged in a (CRBD). For the mean comparisons the Tukey $(\mathrm{P} \leq 0.05 \%)$ test was used. To obtain the profitability of the treatments the Benefit-Cost Relationship was used. The consumption of gramineous $(\mathrm{g})$, consumption of food $(\mathrm{g})$, gain of weight $(\mathrm{g})$, nutritious conversion, live weigh $(\mathrm{g})$ and yield to the channel (\%) was evaluated. The male guinea pigs presented the major $(\mathrm{P}<0.05)$ consumption of gramineous, gain of weight and alive weight (81.65 $\mathrm{g} \mathrm{day}^{-1}$ of MS; $8.66 \mathrm{~g} \mathrm{animal}^{-1}$ day $^{-1} ; 827.7$ $\mathrm{g}$, respectively). The gramineous of more consumption $(\mathrm{P}<0.05)$ was the saboya grass $\left(99.31 \mathrm{~g}\right.$ animal $^{-1}$ day $^{-1}$ of MS). However it shows the less efficient (13.82) nutritious conversion $(\mathrm{P}<0.05)$. The biggest gain of weight $(\mathrm{P}<0.05)$ was registered by the treatment based on leaf of corn $\left(9.16 \mathrm{~g} \mathrm{animal}^{-1}\right.$ day $\left.^{-1}\right)$. The yield to the channel $(\mathrm{P}>0.05)$ was not affected by the effect of the consumption of gramineous. The biggest profitability was registered by the male guinea pigs fed with leaf of corn + balanced $(52.4 \%)$.

Key words: Gramineous, forage, feeding, guinea pigs

\section{INTRODUCCIÓN}

$\mathrm{L}$ a cría y explotación de esta especie animal se la realiza especialmente en la región Interandina, sin el uso de recursos técnicos adecuados, descuidándose aspectos importantes como la alimentación, manejo, sanidad, genética, etc. (Biblioteca La Chacra 1986).

Para contribuir a la cría intensiva de esta especie es necesario aprovechar su condición de herbívoro y evaluar alternativas alimenticias de bajo costo y de fácil adquisición, como las gramíneas, que al combinarlas con una mínima cantidad de balanceado (suplemento) se

Recibido: Junio, 2008. Aceptado: Enero, 2009.

Publicado como ARTÍCULO en Ciencia y Tecnología 2: 25-28. 2009. logra cubrir los requerimientos nutritivos en cada una de las fases por las que atraviesa (cría, recría, engorde, lactancia y gestación) incrementando significativamente su rendimiento.

Los pastos en los cuyes tienen vital importancia ya que ofrecen vitamina $\mathrm{C}$, y a la vez es un vehículo de aporte hídrico. Los cuyes poseen la habilidad para aprovechar los pastos y forrajes debido al volumen del ciego y la flora bacterial allí desarrollada y que tienen como función degradar los alimentos fibrosos y groseros (Caycedo, 1993 y Sarría, 1990).

En el litoral ecuatoriano el pasto saboya $(\mathrm{Pa}-$ 
nicum maximun Jack), el maíz (Zea Mays), y la caña de azúcar (Saccharum officinarum L) son las gramíneas de mayor frecuencia en nuestras condiciones ambientales, ya sean cultivadas o espontáneas proporcionan una elevada producción de biomasa por hectáreas de excelente calidad, utilizada eficientemente en la alimentación animal (INIAP, 1992).

\section{Materiales y Métodos}

T a presente investigación se ejecutó en la granja ـ"San Carlos” localizada en el km 3 vía La Maná San Juan, en el Cantón La Maná; ubicado al Sur - Este de la provincia de Cotopaxi, su ubicación geográfica es de $0{ }^{\circ} 45^{\prime} 35^{\prime}$ ' de latitud Sur y $7^{\circ} 09^{\prime} 32$ ' de longitud Oeste a una altura de $240 \mathrm{msnm}$. El trabajo de campo duró 56 días. Con una temperatura promedio de $24.22^{\circ} \mathrm{C}$, una humedad relativa, $89.7 \%$, y una precipitación anual de 2948.9 milímetros.

\section{Tratamientos y diseño experimental}

De la combinación de los factores se obtuvieron los siguientes tratamientos:

$\boldsymbol{s} \mathbf{1} \boldsymbol{g} \mathbf{1}=$ Hembras + pasto saboya + balanceado.

$\boldsymbol{s} \mathbf{1} \mathbf{g}$ = Hembras + follaje de maíz + balanceado.

s1 $\mathbf{g} 3$ = Hembras + follaje de caña de azúcar + balanceado.

s2 $\mathbf{g} \mathbf{1}=$ Machos + pasto saboya + balanceado.

$\boldsymbol{s} \mathbf{g} \mathbf{2}=$ Machos + follaje de maíz + balanceado.

s2 $\mathbf{g} 3$ = Machos + follaje de caña de azúcar + balanceado.

Se aplicó un arreglo factorial 2 (sexo) x 3 (gramíneas) con 4 repeticiones dentro de un diseño de bloques completos al Azar (DBCA). La unidad experimental estuvo conformada por dos animales. Para determinar las diferencias estadísticas entre medidas se aplicó la prueba de rangos múltiples de Tukey $(\mathrm{P}<$ 0.05). Se evaluó: consumo de gramíneas (g), consumo de alimento $(\mathrm{g})$, ganancia de peso $(\mathrm{g})$, conversión alimenticia, peso vivo $(\mathrm{g})$ y rendimiento a la canal $(\%)$.

\section{Resultados y Discusión}

L os resultados de las variables evaluadas se detallan en los cuadros 1 y 2 . El mayor consumo de gramínea lo registraron los cuyes machos (4572.5 g lo que representa un consumo animal ${ }^{-1}$ día $^{-1}$ de $81.65 \mathrm{~g}$ de MS). De igual manera estos registraron el mayor consumo de alimento (forraje + balanceado, 5292.7 g MS); ganancia de peso (484.9 g), conversión alimenticia (11.57) y rendimiento a la canal $(64.6 \%)$.

Los valores obtenidos para el consumo de alimento y ganancia de peso superan a los reportados por Proaño (1993), quien al evaluar el efecto de la retama verde (Sphaero carpa) en sustitución $(0 ; 10,20$ y 30 $\%$ ) de la alfalfa (Medicago sativa) sobre el sexo en las etapas de crecimiento y engorde (14-90 días) registró los siguientes valores 3519; $411 \mathrm{~g}$, en su orden.

Sin embargo, los valores registrados en la presente investigación para la ganancia de peso; conversión alimenticia y rendimiento a la canal son inferiores a los reportados por Padilla (1990), quien al evaluar niveles de gallinaza en la alimentación de cuyes en las fases de gestación, lactancia, crecimiento y engorde, concluyó que los cuyes machos presentaron mayor ventaja con relación a las hembras registrando los siguientes valores $519 \mathrm{~g} ; 7.02$ y $68.01 \%$, respectivamente.

La gramínea de mayor consumo fue el pasto saboya (5561.8 g lo que representa un consumo animal ${ }^{-1}$ día $^{-1}$ de $99.31 \mathrm{~g}$ de MS). Este mayor consumo puede deberse a que esta gramínea es de menor calidad con respecto a las hojas de maíz y de caña, por lo tanto el animal incrementa el consumo para cubrir sus requerimientos nutricionales.

La mayor ganancia de peso lo registró el tratamiento en base a la hoja de maíz + balanceado (512.85 y $852.5 \mathrm{~g}$ ) mientras tanto los tratamientos bajo el efecto del consumo de la hoja de maíz y caña de azúcar +balanceado reportaron la conversión alimenticia más eficiente $(10.7$ y $10.6 \%)$, esto se debe posiblemente a la mejor calidad del alimento suministrado. El rendimiento a la canal no se vio afectado por el efecto del consumo de gramínea, sin embargo, el tratamiento en base a pasto saboya reporto el mayor rendimiento a la canal $(65.2 \%)$.

Los resultados de la presente investigación respecto a la ganancia de peso y conversión alimenticia superan a los reportados por Cevallos (1995), quien al evaluar el efecto del cubo multinutricional suplementados con pasto saboya y elefante en el crecimiento y engorde de cuyes peruanos, reportó una ganancia, y conversión alimenticia de $5.96 \mathrm{~g}_{\text {animal }}^{-1}$ día $^{-1}$ y 12.6 para el tratamiento en base a pasto elefante + cubo multinutricional.

De igual manera la ganancia de peso de la presente investigación (9.15 $\left.\mathrm{g}_{\text {animal }}{ }^{-1} \mathrm{dí}^{-1}\right)$ supera a las reportadas por Ante (2002) y Plaza (2001), quienes al evaluar el efecto del ramio (Bohemeria nivea Gaud), gramíneas y leguminosas en el engorde de cuyes reportaron ganancias de $8.68 \mathrm{~g}$ para el tratamiento en base a pasto saboya + balanceado. Sin embargo, el rendimiento a la canal fue inferior a los reportados por Cevallos (1995); Ante (2002) y Plaza (2001) quienes registraron 
rendimientos del $72.54 ; 67.50 ;$ y $71.64 \%$, en su orden. Además superan a los reportados por Oleas (1982) y con los de Fernández (1984), citados por Cayancela (1993), que obtuvieron ganancias diarias de 6.11 y $6.43 \mathrm{~g}$ ani$\mathrm{mal}^{-1} \mathrm{dia}^{-1}$ respectivamente, utilizando diferentes niveles de gallinaza y quinuharina. Sin embargo la ganancia de peso se vio superada por la reportada por Cayancela (1993), quien al utilizar la harina de retama $(5 ; 10 ; 15$ y $20 \%$ de inclusión en la dieta) reportó una ganancia diaria de $9.65 \mathrm{~g}$ animal $^{-1}$ dìa $^{-1}$ con el $5 \%$ de inclusión y por los de Caycedo (1992), quien reporta ganancias y conversiones de $10.0 \mathrm{~g}_{\text {animal }}{ }^{-1}$ dìa $^{-1}$ y 5.01 , en cuyes mejorados.

La mayor rentabilidad la registraron los cuyes machos alimentados con hoja de maíz + balanceado (52.4\%). La rentabilidad registrada en la presente investigación supera a la obtenida por Plaza (2001) quien al evaluar los efectos de gramíneas y leguminosas en el engorde de cuyes reporta una rentabilidad del $47.3 \%$.

\section{Conclusiones}

T os resultados obtenidos en la presente investigación unos permiten llegar a las siguientes conclusiones.

Los cuyes machos registraron el mayor comportamiento en las variables consumo de gramínea y ganancia de peso.

El pasto saboya fue la gramínea de mayor consumo, sin embargo, registra la conversión alimenticia menos eficiente.

La hoja de caña de azúcar fue la gramínea de menor consumo. Además registró la menor ganancia de peso.

Los cuyes machos alimentados con hoja de maíz registraron la mayor ganancia de peso y rentabilidad.

El rendimiento a la canal no se vio afectado por el efecto del sexo ni por el consumo de gramíneas.

\section{Literatura Citada}

Ante, L. 2002. Efecto del ramio Bohemeria nivea Gaud en la alimentación de cuyes peruanos (Cavia porcellus L) en la etapa de engorde. Tesis. Ing. Zoot. Facultad de Ingeniería Zootécnica, Universidad Técnica Estatal de Quevedo (UTEQ). Quevedo, Los Ríos, Ecuador. 53-55 p.
BIBLIOTECA LA CHACRA 1986. Producción y crianza del Cuy. Editorial Mercurio S.A. 77-79 p.

Caycedo, V. 1992. Investigaciones en cuyes. III Curso Latinoamericano de Producción de Cuyes. Lima, Perú, UNA - La Molina. Lima, Perú.

Caycedo, V. 1993. Efecto de la frecuencia de suministro de forraje y suplemento concentrado en los rendimientos productivos del cuy Cavia porcellus L. revista Latinoamericano de investigaciones en pequeños herbívoros no rumiantes $60-67 \mathrm{p}$.

Cayancela, A. 1993. Efecto de varios niveles de harina de retama en la alimentación de cuyes en las etapas de crecimiento-engorde. IV Congreso Latinoamericano de Cuyecultura. Riobamba, Ecuador. pp. 35-37.

Cevallos, A. 1995. Utilización del cubo multinutricional en el crecimiento y engorde de cuyes peruanos Cavia porcellus L. Tesis Ing. Zoot. Escuela de Ingeniería Zootécnica Universidad Técnica Estatal de Quevedo. (UTEQ). Quevedo, Los Ríos, Ecuador. pp. 66-74.

INIAP, 1992. Instituto Nacional de Investigaciones Agropecuarias. Manual de pastos tropicales Quito, Ecuador 6 - 13 p.

Padilla, A. 1990. Utilización de niveles de gallinaza en la alimentación de cuyes peruanos mejorados en gestación, lactancia, crecimiento y engorde. Tesis de Grado. Facultad de Ingenieria Zootécnia. Escuela Superior Politécnica del Chimborazo (ESPOCH). Riobamba, Ecuador. p 55.

Plaza, Z y Suárez, L. 2001. Alimentación de cuyes peruanos mejorados Cavia porcellus L con asociación de gramíneas-leguminosas. Tesis Ing. Zoot. Escuela de Ingeniería Zootécnica, Facultad de Ciencias Pecuarias. Universidad Técnica Estatal de Quevedo (UTEQ). Quevedo, Los Ríos, Ecuador. pp.52.

Proaño, J. 1993. Efecto de la retama verde Sphaero car$p a$ en sustitución de la alfalfa en la alimentación de cuyes mejorados. Tesis Ing. Zoot. Facultad de Ingeniería. Zootécnica de la Escuela Politécnica de Chimborazo (ESPOCH). Riobamba, Ecuador. p.50

Sarría, JB. 1990. La crianza de cuyes tecnología básica y problema y alternativas de la alimentación de los animales menores Universidad Técnica Nacional Agraria. Departamento de producción Animal Lima, Perú. p.9-11. 
Cuadro 1. Efecto simple del sexo y tres gramíneas tropicales para las variables consumo de forraje, consumo de alimento, ganancia de peso, conversión alimenticia y rendimiento a la canal, en el engorde de cuyes sexados (Cavia porcellus Linnaeus). La Maná, 2007

\begin{tabular}{lccccc}
\hline \multicolumn{1}{c}{ Factores } & $\begin{array}{c}\text { Consumo de } \\
\text { forraje (g) }\end{array}$ & $\begin{array}{c}\text { Consumo de } \\
\text { alimento (g) }\end{array}$ & $\begin{array}{c}\text { Ganancia de } \\
\text { peso (g) }\end{array}$ & $\begin{array}{c}\text { Conversión } \\
\text { Alimenticia }\end{array}$ & $\begin{array}{c}\text { Rendimiento a } \\
\text { la canal (\%) }\end{array}$ \\
\hline a) Sexo & & & & & \\
\hline Hembras & $4331,9 \mathrm{~b}$ & 5047,2 & $447,5 \mathrm{~b}$ & 11,8 & 64,1 \\
Machos & $4572,5 \mathrm{a}$ & 5292,7 & $484,9 \mathrm{a}$ & 11,6 & 64,6 \\
\hline b) Gramíneas & & & & & \\
\hline Pasto Saboya & $5561,8 \mathrm{a}$ & $6272,8 \mathrm{a}$ & $478,5 \mathrm{~b}$ & $13,8 \mathrm{a}$ & 65,2 \\
Hoja de Maíz & $4656,9 \mathrm{~b}$ & $5380,4 \mathrm{~b}$ & $521,9 \mathrm{a}$ & $10,7 \mathrm{~b}$ & 64,8 \\
Hoja de Caña de azúcar & $3188,1 \mathrm{c}$ & $3856,6 \mathrm{c}$ & $398,3 \mathrm{c}$ & $10,6 \mathrm{~b}$ & 63,2 \\
\hline
\end{tabular}

a. b.c: Letras desiguales dentro de columnas y grupo de factores indican diferencias significativas $(\mathrm{P}<0,05)$

Cuadro 2. Análisis económico (USD) de la evaluación de gramíneas tropicales en el engorde de cuyes sexados mejorados (Cavia porcellus Linnaeus). La Maná, 2007

\begin{tabular}{cccccc}
\hline \multicolumn{5}{c}{ Sexo } \\
\hline $\begin{array}{c}\text { Pasto } \\
\text { saboya }+ \\
\text { balanceado }\end{array}$ & $\begin{array}{c}\text { Follaje de } \\
\text { maíz }+ \\
\text { balanceado }\end{array}$ & $\begin{array}{c}\text { Follaje de caña } \\
\text { de azúcar }+ \\
\text { balanceado }\end{array}$ & $\begin{array}{c}\text { Pasto } \\
\text { saboya }+ \\
\text { balanceado }\end{array}$ & $\begin{array}{c}\text { Follaje de } \\
\text { maíz }+ \\
\text { balanceado }\end{array}$ & $\begin{array}{c}\text { Follaje de caña } \\
\text { de azúcar }+ \\
\text { balanceado }\end{array}$ \\
\hline 30,332 & 33,409 & 28,802 & 33,103 & 37,625 & 30,995 \\
24,750 & 24,679 & 24,553 & 24,768 & 24,696 & 24,578 \\
5,582 & 8,730 & 4,249 & 8,335 & 12,929 & 6,417 \\
$\mathbf{2 2 , 6}$ & $\mathbf{3 5 , 4}$ & $\mathbf{1 7 , 3}$ & $\mathbf{3 3 , 7}$ & $\mathbf{5 2 , 4}$ & $\mathbf{2 6 , 1}$ \\
\hline
\end{tabular}

